\title{
Dynamic DC-link Voltage Adaptation for Thermal Management of Traction Drives
}

\author{
Joris Lemmens and Johan Driesen \\ Dept. of Electrical Engineering ESAT-ELECTA \\ Katholieke Universiteit Leuven \\ B-3001 Leuven, Belgium
}

\author{
Piet Vanassche \\ TRIPHASE NV \\ B-3001 Leuven, Belgium
}

\begin{abstract}
Power density and reliability specifications for motor drives in traction applications are getting increasingly stringent. The main challenge in meeting these conflicting requirements, is managing heat dissipation. A drive's peak torque rating is limited by switching device temperatures which must be kept below critical values at all times for the sake of reliability, preferably without major hardware adaptations. In this challenge lies a large potential for advanced control algorithms. This paper proposes a PMSM drive control strategy which combines active thermal management with dynamic DC-link voltage adaptation. The bus voltage level is adjusted to the required PMSM terminal voltage in each operating point. Doing so, switching losses can be reduced at low speed by lowering the bus voltage. At high speed, the voltage level is boosted and field-weakening operation and the associated additional losses are avoided. An 11 kW PMSM drive, with an active front-end controlling the bus voltage, is used as a test setup to mimic a series-hybrid drivetrain. Compared to a fixed DC-link voltage, efficiency maps show a significant inverter loss reduction at low speed. This results in lower switching device temperatures which in turn allows a higher peak torque rating.
\end{abstract}

\section{INTRODUCTION}

In the challenging thermal environment of automotive applications, the ever increasing power density and reliability requirements for traction drives push the limits of current technology. Power semiconductor devices are known to be the most vulnerable components in a drivetrain [1]. Due to temperature gradients and differing coefficients of thermal expansion, internal connections in the power module undergo thermo-mechanical strain during power or temperature cycling. This can invoke a number of failure mechanisms such as solder fatigue and bond-wire lift-off [2]. Lifetime estimation models inversely relate the number of cycles to failure to the mean junction temperature and temperature cycling amplitude [2][4]. Hence, adequate thermal management is indispensable to meet the conflicting future requirements on power density and reliability. Increasing a drive's peak power rating requires an equivalent improvement of heat dissipation capability to keep component temperatures within a safe operating area. Unfortunately, this often goes to the expense of additional weight, volume and cost. Another approach is to concentrate on the heat sources, rather than on the heat sinks. Reducing drivetrain losses not only increases vehicle mileage, but also allows for a higher power rating and a more compact machine and converter design. This can be achieved through hardware improvements, but a large potential lies in the control strategy as well. Advanced algorithms incorporating efficiency optimization and active thermal management can improve performance figures of drivetrains significantly without adding substantial cost. Such control strategies should exploit all available control variables to optimize the operating regime. This comes down to minimizing the losses, while maximizing torque output without exceeding electro-thermal boundaries. Previous work [5] proposed an active thermal management strategy for PMSM traction drives. It is based on real-time temperature estimation of switching devices and stator windings. When a component approaches a critical temperature, active loss control prevents overheating and excessive power cycling by means of switching frequency reduction and current limiting. Furthermore, the dq-current setpoint calculation is tackled as a constraint optimization problem. Hence, torque and efficiency are maximized inside the temperature and voltage constraints in every operating point.

The goal of this paper is to extend the control strategy of [5] with dynamic DC-link voltage adaptation, providing an additional degree of freedom to reduce losses. At low speed, the voltage needed to control the motor is much lower than at rated speed due to the speed-dependent back electromotive force. Hence, the switching losses in the inverter can be reduced significantly by lowering the DC-link voltage [6]. When speed increases, flux weakening is necessary from the point where the maximum terminal voltage, given the bus voltage and modulation strategy, is reached. The additional negative d-axis current needed to counteract the permanent magnet flux will cause additional conduction losses and efficiency will decrease rapidly. By boosting the DC-link voltage, fluxweakening operation can be avoided or delayed [7], [8]. Variable DC-link control was already successfully implemented in commercialized battery-powered vehicles [9]. A drawback however is the DC-DC converter which adds to the complexity and cost of the system. Hence, a tradeoff with the efficiency gain and other benefits should be made [10].

The analysis in this paper assumes a series-hybrid topology, which has the inherent ability to adjust DC-link voltage by means of the PFC (power factor corrector) between the internal combustion engine (ICE) driven generator and the DC-bus. The main goal of this paper is to analyze the added value of dynamic DC-link voltage adaptation regarding thermal management for this topology. 


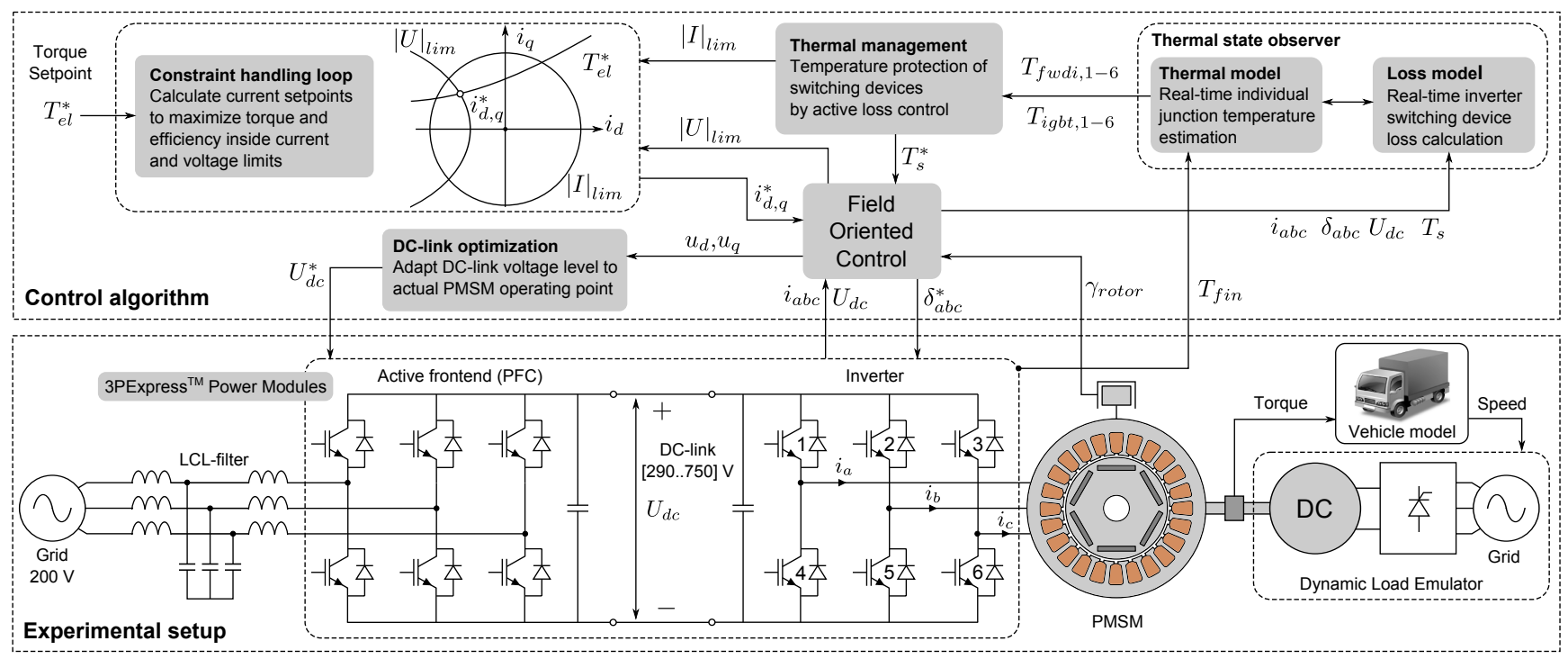

Fig. 1. Block diagram of the proposed control algorithm and experimental setup.

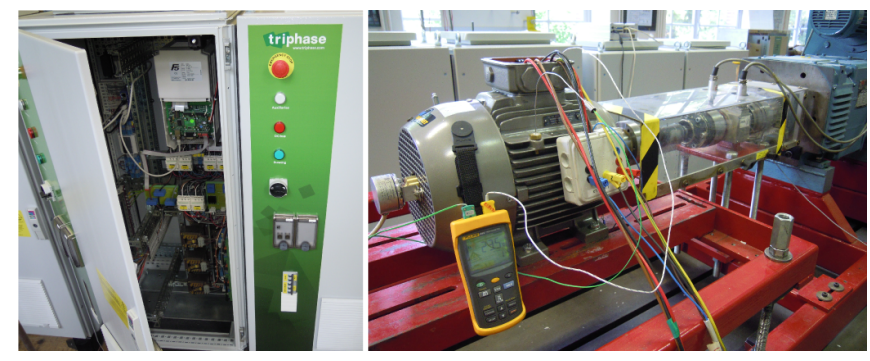

Fig. 2. Experimental setup: 3PExpress ${ }^{\mathrm{TM}}$ Rapid Prototyping Platform [11] controlling an $11 \mathrm{~kW}$ PMSM and a DC-machine for dynamic load emulation.

\section{EXPERIMENTAL SETUP}

Fig. 1 gives an overview of the experimental setup and control algorithm. The setup mimics a series-hybrid drivetrain topology (without considering battery storage), using modular power electronic converters as shown in Fig. 2. The vehicle's ICE-driven synchronous generator is represented by the grid which is interfaced through a $17 \mathrm{kVA}$ active frontend. This power module performs power factor correction, facilitates regenerative braking and dynamic DC-bus voltage control. The lower bound of the control range is determined by the grid voltage; $U_{d c, \min }=\sqrt{2} U_{\text {grid }}$ with space vector modulation (SVM). However, reducing the grid voltage also affects the frontend's power rating. Therefore, line voltage was lowered to $200 \mathrm{~V}$ providing a relatively large DC control range of 290 to $750 \mathrm{~V}$ while maintaining a sufficient power rating (approx. $10 \mathrm{kVA})$. A second $17 \mathrm{kVA}$ power module controls the $11 \mathrm{~kW}$ PMSM which has a rated torque of $70 \mathrm{Nm}$ at $1500 \mathrm{RPM}$. This machine is mounted on a test bench including a torque transducer and a DC-machine for dynamic load emulation. The control algorithm is implemented in Matlab/Simulink ${ }^{\mathrm{TM}}$, using the Triphase 3PExpress ${ }^{\mathrm{TM}}$ rapid prototyping platform [11] for interaction with both power modules.

\section{Drivetrain Control Strategy}

The objective of the control strategy in Fig. 1 is optimizing drivetrain performance, i.e. maximizing torque output and efficiency within electro-thermal constraints.

PMSM electromagnetic torque $T_{e l}$ is given by:

$$
T_{e l}=p i_{q}\left[\Psi-\left(L_{q}-L_{d}\right) i_{d}\right]
$$

with $p=3$ the number of pole pairs, $i_{d}$ and $i_{q}$ the direct and quadrature motor currents, $\Psi=0.7885 \mathrm{Vs}$ the permanent magnet flux linkage, $L_{d}=10 \mathrm{mH}$ the d-axis inductance and $L_{q}=19 \mathrm{mH}$ the q-axis inductance. With $R_{s}=0.336 \Omega$ the stator resistance and $\omega_{e l}$ the angular electrical frequency, the steady state motor voltages in the dq-framework are:

$$
\left\{\begin{array}{l}
u_{d}=R_{s} i_{d}-\omega_{e l} L_{q} i_{q} \\
u_{q}=R_{s} i_{q}+\omega_{e l} L_{d} i_{d}+\omega_{e l} \Psi
\end{array}\right.
$$

\section{A. Motor and Inverter Losses}

The majority of motor losses are due to copper loss $P_{C u}$ and iron loss $P_{F e}$, given in equations (3) and (4) respectively with $R_{F e}$ the equivalent iron loss resistance.

$$
\begin{gathered}
P_{C u}=R_{s}\left(i_{d}^{2}+i_{q}^{2}\right) \\
P_{F e}=\frac{\left(u_{d}-R_{s} i_{d}\right)^{2}+\left(u_{q}-R_{s} i_{q}\right)^{2}}{R_{F e}} \\
R_{F e}=2.1182 \omega_{e l}+42.427
\end{gathered}
$$

Losses in the inverter can be subdivided into switching and conduction losses in the IGBTs and freewheeling diodes (FWDIs). The conduction losses in each device, averaged over one electrical period, are given by [2]:

$$
\bar{P}_{c, i g b t}=\frac{1}{2 \pi} \int_{0}^{\pi} V_{c e}\left(i, T_{j}\right) \cdot \hat{i} \sin (x) \frac{1+\hat{\delta} \operatorname{svm}(x+\varphi)}{2} d x
$$




$$
\bar{P}_{c, f w d i}=\frac{1}{2 \pi} \int_{\pi}^{2 \pi} V_{e c}\left(i, T_{j}\right) \cdot|\hat{i} \sin (x)| \frac{1+\hat{\delta} \operatorname{svm}(x+\varphi)}{2} d x
$$

with $\hat{i}=\sqrt{\frac{2}{3}\left(i_{d}^{2}+i_{q}^{2}\right)}$ the phase current amplitude, $\hat{\delta}$ the modulation index between 0 and 1 , svm the space vector modulation function, $\varphi$ the phase angle and $V_{c e}, V_{e c}$ the current and temperature dependent IGBT and FWDI voltage drop [12]. IGBT switching loss and FWDI reverse recovery loss are given by [2]:

$$
\begin{gathered}
\bar{P}_{s w, i g b t}=\frac{1}{T_{s}} \cdot\left(E_{o n}+E_{o f f}\right) \cdot \frac{1}{\pi} \cdot \frac{\hat{i}}{i_{r e f}} \cdot\left(\frac{U_{d c}}{U_{r e f}}\right)^{1.3} \\
\bar{P}_{r r, f w d i}=\frac{1}{T_{s}} \cdot E_{r r} \cdot\left(\frac{1}{\pi} \cdot \frac{\hat{i}}{i_{r e f}}\right)^{0.6} \cdot\left(\frac{U_{d c}}{U_{r e f}}\right)^{0.6}
\end{gathered}
$$

with $T_{s}$ the switching period, $U_{d c}$ the DC-link voltage and $E_{o n}, E_{o n}, E_{o n}$ the switching energy losses [12] (at reference current $i_{r e f}$ and DC-bus voltage $U_{r e f}$ ).

As all loss components contain a current-dependency, the dq-current setpoint is identified as a first degree of freedom for efficiency optimization and active thermal management. In addition, dynamic adaptation of $U_{d c}$ and $T_{s}$ also provides a way to control drivetrain losses.

\section{B. Constraint Optimal Control}

1) MEPNm Control: According to (1), each torque request $T_{e l}^{*}$ can be met with an infinite number of possible current setpoint combinations $i_{d}^{*}$ and $i_{q}^{*}$. However, practical voltage and current limitations restrict the operating area in the dqcurrent plane. Inside this area, the dq-current combination can be optimized to obtain a desired operating regime, for example unity power factor or maximum torque per ampere (MTPA) control. This paper implements a Maximum Efficiency per $\mathrm{Nm}$ (MEPNm) strategy for minimal overall losses $P_{o}$. In comparison, MTPA minimizes current amplitude and hence only optimizes conduction and copper losses. MEPNm is especially beneficial at elevated speed, when iron losses become substantial [5]. At low speed, the difference between MEPNm and MTPA is negligible. Solving the torque equation (1) to $i_{q}$ and substituting into the overall loss expression (10), results in a single variable $\left(i_{d}\right)$ loss equation. All other loss-determining process variables $\left(U_{d c}, T_{s}, \hat{\delta}, \varphi, \omega_{e l}\right)$ are considered as parameters, equal to the instantaneous (measured) value at each iteration. Finding the MEPNm d-current setpoint $i_{d, o p t}^{*}$ comes down to finding the global minimum of the convex overall loss function. This is done with a Newton iteration adjusting the previous d-current setpoint $i_{d, 0}$ with a step $\mathrm{d} i_{d, o p t}$.

$$
\begin{gathered}
P_{o}=P_{C u}+P_{F e}+6 \cdot\left(\bar{P}_{c, i g b t}+\bar{P}_{c, f w d i}+\bar{P}_{s w, i g b t}+\bar{P}_{r r, f w d i}\right) \\
\mathrm{d} i_{d, o p t}=-\left.\frac{\frac{\mathrm{d} P_{o}}{\mathrm{~d} i_{d}}}{\frac{\mathrm{d}^{2} P_{o}}{\mathrm{~d} i_{d}^{2}}}\right|_{i_{d}=i_{d, 0}} \\
i_{d, o p t}^{*}=i_{d, 0}+\mathrm{d} i_{d, o p t}
\end{gathered}
$$

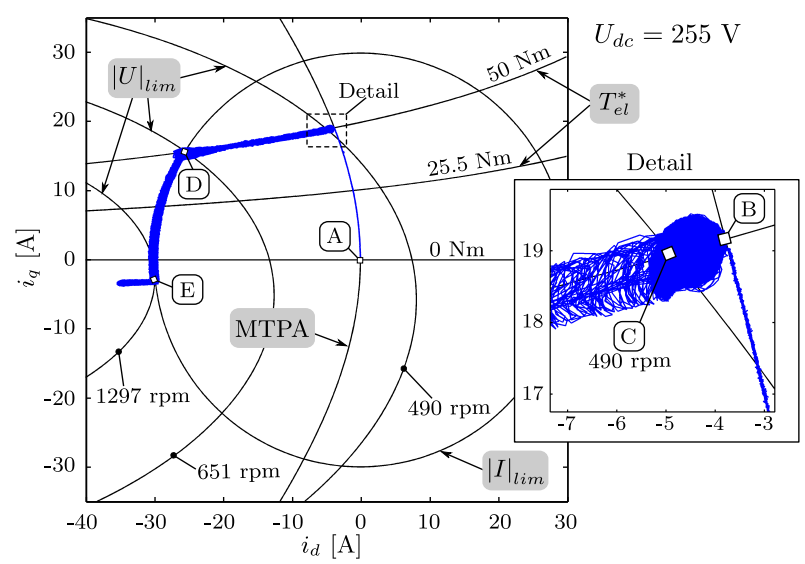

Fig. 3. Constraint Optimal Control: PMSM dq-current operating points.

2) Constraint Optimization: A constraint optimization algorithm, based on [13], generates optimal dq-current setpoints $i_{d}^{*}$ and $i_{q}^{*}$ which minimize disturbance on $T_{e l}^{*}$ and $i_{d, o p t}^{*}$, given the maximum output voltage $|U|_{\text {lim }}$ and current limit $|I|_{\text {lim }}$. The voltage constraint considering SVM (13) follows from the DC-link voltage and a small control margin $\Delta U=15 \mathrm{~V}$. The current constraint $|I|_{\text {lim }}$ can either be fixed to the inverter's maximum rating [14] or be imposed by the active thermal management loop (see further).

$$
|U|_{l i m}=\frac{U_{d c}}{\sqrt{2}}-\Delta U
$$

Without going into detail, the strategy in [13] incorporates a priority-based algorithm:

1) $|I|=\sqrt{i_{d}^{2}+i_{q}^{2}} \leq|I|_{\text {lim }}$ The current setpoint must be inside the current limit circle to protect components from thermally induced failure.

2) $|U|=\sqrt{u_{d}^{2}+u_{q}^{2}} \leq|U|_{\text {lim }}$ The current setpoint must be inside the voltage limit ellipse to prevent overmodulation and eventually loss of PMSM control. Apply field weakening when voltage limit is reached.

3) Try to deliver the requested torque $T_{e l}^{*}$. If this is inhibited by current and/or voltage constraints, apply a current setpoint as close as possible to the equi-torque line.

4) Apply the optimal MEPNm d-current setpoint $i_{d, o p t}^{*}$.

Fig. 3 shows the resulting dq-currents, recorded during a test run. First, torque request is gradually increased from 0 to 50 $\mathrm{Nm}$ with a locked rotor. This corresponds to a shift of the operating point from A to B along the MTPA-curve. Next, the rotor is released and the motor starts accelerating with constant torque. As iron loss increases with speed, the MEPNm strategy gradually applies field weakening so the operating point is shifted from B to C. At 490 RPM, the voltage limit is reached. As speed increases further, field weakening shifts the operating point from $\mathrm{C}$ to $\mathrm{D}$ along the $50 \mathrm{Nm}$ equi-torque line. After reaching the $30 \mathrm{~A}$ current limit in $\mathrm{D}$, the operating point shifts along the current limit circle to E. However, this implies a reduction of output torque. In E, the maximum allowable speed 

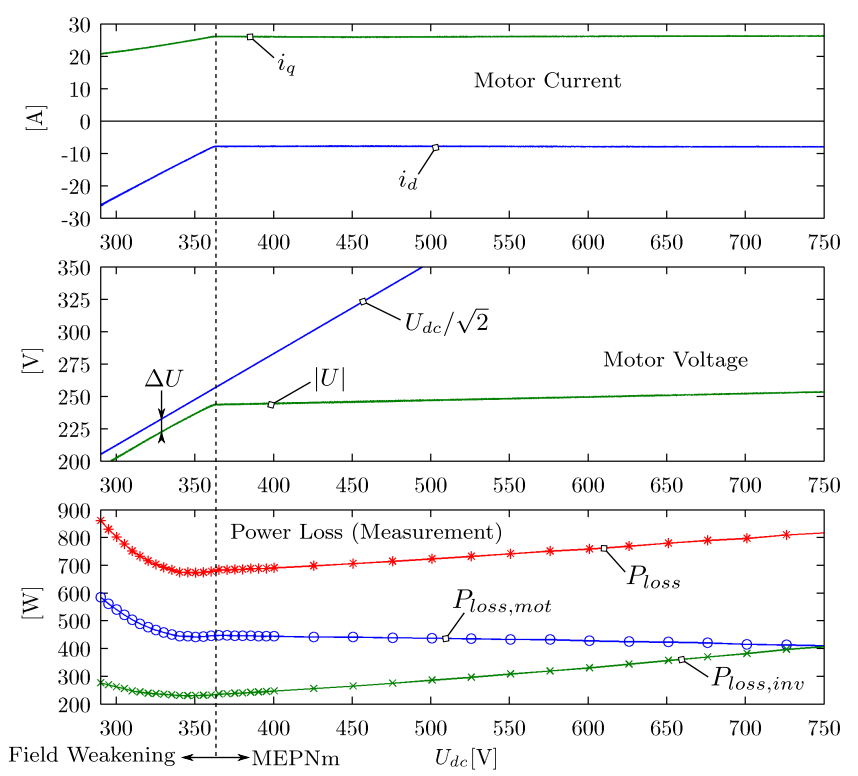

Fig. 4. Measured motor current, voltage and power loss at constant load (63.5 Nm $800 \mathrm{RPM}$ ) as a function of DC-bus voltage.

according to the current and voltage limit is reached. Operation beyond this point is not allowed because it implies a violation of the current (i.e. temperature) limit.

\section{Dynamic DC-link Voltage Adaptation}

The active frontend facilitates dynamic adaptation of $U_{d c}$ between 290 and 750 V. A lower DC-link voltage can reduce switching losses according to (8) and (9). However, from the point where the voltage limit is reached, this advantage is cancelled out because the constraint optimization loop will increase field weakening current and move away from the optimum $i_{d, o p t}^{*}$. This is illustrated by Fig. 4 showing inverter, motor and total power loss at constant load as a function of $U_{d c}$. Losses were measured by means of a torque/speed transducer and two Voltech PM3000 power analyzers, one at the DClink and one at the motor terminals. An optimum is reached at the boundary between MEPNm and field-weakening mode. Hence, the straightforward optimization strategy generates a setpoint $U_{d c}^{*}$ according to (14). Voltages $u_{d}, u_{q}$ are available from the PI current control loops. Given the practical voltage limitations, this results in an optimized DC-link voltage in each speed-torque operating point as shown in Fig. 5.

$$
U_{d c}^{*}=\sqrt{2}\left[\sqrt{u_{d}^{2}+u_{q}^{2}}+\Delta U\right]
$$

\section{Active Thermal Management}

1) Motivation: Industrial drives mainly operate at relatively high fundamental frequencies compared to the thermal time constants of power switching devices. This implies that the variation of junction temperature with load current during a modulation cycle is sufficiently small to be neglected. However, traction drives operate for prolonged periods at high torque and low speed, for example in urban traffic. When

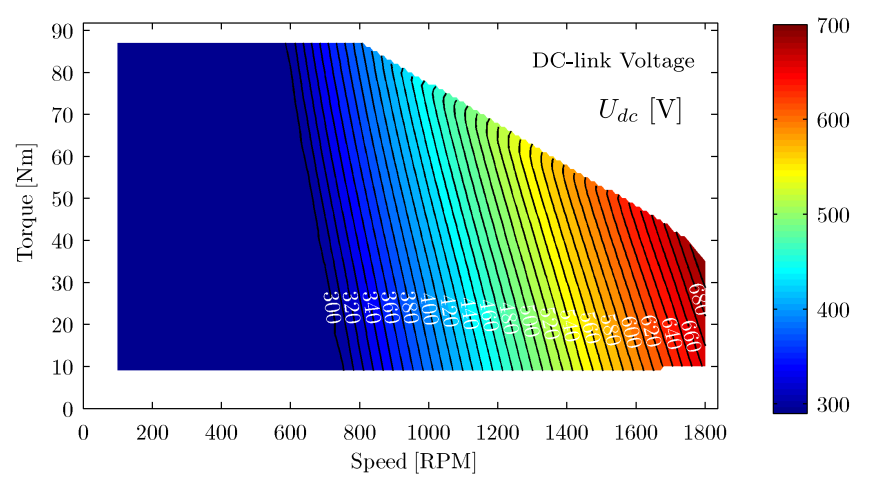

Fig. 5. DC-link voltage (measurement) is adapted within the 290-750 V control range according to the actual speed-torque operating point.

modulation frequency decreases $(<2 \ldots 5 \mathrm{~Hz})$ at equal torque output, power cycling becomes more apparent due to the low pass filter characteristic of the junction to case transient thermal impedance. Switching device temperatures show a larger fluctuation and reach a higher peak value, despite equal mean losses. In addition, power modules are generally optimized for inverter duty with the assumption of a modulation index greater than 0.5 . For this type of duty, the majority of load is on the IGBTs. Hence, they are provided with a larger chip surface, i.e. smaller junction to case thermal resistance, compared to the FWDIs. The undersized diodes can become problematic at low speed operation, i.e. when dominant load is on the FWDIs (small modulation index). For a PMSM drive operating at standstill torque, the single diode which continuously conducts the largest DC phase current is most prone to failure. As indicated in the introduction, reliability is a function of junction temperature cycle and amplitude. A common approach to keep component temperatures within a safe operating area is setting a fixed current limit to the motor controller, as specified in the inverter's datasheet [14]. This limit should account for the previously described worst case load conditions and the highest anticipated ambient temperature. However, this also means a loss of performance at non-worst-case load and ambient conditions, i.e. torque is restricted without thermal limits actually being reached. Active thermal management [5] can offer a significant performance improvement because it sets a dynamic current limit based on actual temperature feedback. Hence only limiting torque when thermal boundaries are approached.

2) Real-time Switching Device Temperature Estimation: Fig. 6 shows the internal layout of the three-phase IGBT power module in the inverter. It consists of six IGBT-diode pairs soldered to a direct bonded copper (DBC) substrate. The substrate is soldered to a copper base plate which is mounted on a heatsink. In order to estimate the junction temperatures, the device losses are supplied to thermal equivalent circuits. Switching and conduction losses, averaged over one fundamental electric period, were already described by equations (6) to (9). However, the thermal management strategy requires real-time temperature feedback of all twelve junctions 

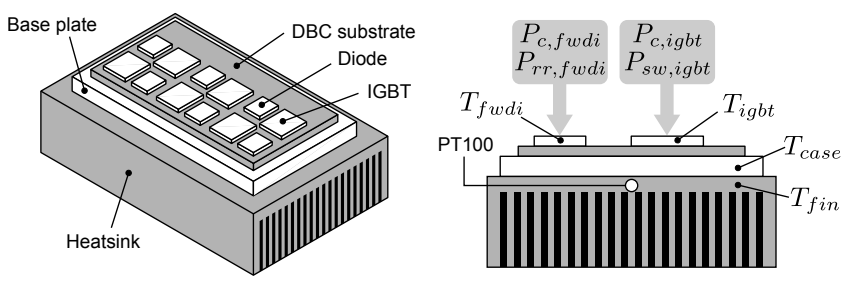

Fig. 6. Layout of a three-phase power module mounted on a heatsink.

individually. Therefore, the thermal model includes power loss calculations averaged over one switching timecycle $T_{s}$. Conduction loss in each component is calculated with (15) and (16), with $\delta$ the duty cycle $(-1 \leq \delta \geq 1), k=1$ for the devices at the upper and $k=-1$ for devices at the lower side of the inverter bridge. Switching loss and reverse recovery loss are given by (17) and (18). Note that dependencies of device properties on current and temperature are stored as polynomials fitted on data sheet graphs [12].

$$
\begin{gathered}
P_{c, i g b t}=V_{c e}\left(i_{i g b t}, T_{i g b t}\right) \cdot i_{i g b t} \frac{1+k \delta}{2} \\
P_{c, f w d i}=V_{e c}\left(i_{f w d i}, T_{f w d i}\right) \cdot i_{f w d i} \frac{1+k \delta}{2} \\
P_{s w, i g b t}=\frac{1}{T_{s}} \cdot\left[E_{o n}\left(i_{i g b t}\right)+E_{o f f}\left(i_{i g b t}\right)\right] \cdot\left(\frac{U_{d c}}{U_{r e f}}\right)^{1.3} \\
P_{r r, f w d i}=\frac{1}{T_{s}} \cdot E_{r r}\left(i_{f w d i}\right) \cdot\left(\frac{U_{d c}}{U_{r e f}}\right)^{0.6}
\end{gathered}
$$

Fig. 7 shows the thermal model consisting of twelve separate Cauer equivalent circuits, one for each IGBT and FWDI, with the assumption of a uniform base plate temperature. Power module manufacturers usually provide a transient junction-case thermal impedance curve from which the $\mathrm{R}$ and $\mathrm{C}$ elements can be extracted. Inputs are the device's switching and conduction losses and the baseplate temperature $T_{\text {case }}$ from the underlying case-ambient model. The case-heatsink interface $R_{c f}$ is assumed purely resistive. Heatsink thermal capacitance $C_{f i n}$ and resistance $R_{f a}$ were determined by means of a step-response test. Inputs are the total power module losses $\sum P_{\text {loss }}$ and the ambient temperature $T_{a m b}$. In addition, the model offers the possibility to include a heatsink temperature sensor $T_{\text {fin }}$. By combining both model and measurement in a Kalman filter structure, junction temperature estimation accuracy can be improved considerably compared to an entirely model-based approach. Fig. 8 shows the losses and temperatures of IGBT-FWDI pair 1 as calculated by the model in real-time, at $67 \mathrm{Nm}$ and $1000 \mathrm{RPM}$.

3) Active Loss Control: Fig. 9 gives a block diagram of the active thermal management strategy. When the highest instantaneous IGBT or FWDI temperature approaches the maximum allowable value $T_{j, l i m}$, switching frequency is reduced first from the nominal value of $10 \mathrm{kHz}$ by means of a four-level hysteresis controller. This reduces switching losses according to (17) and (18), without affecting output torque. In order

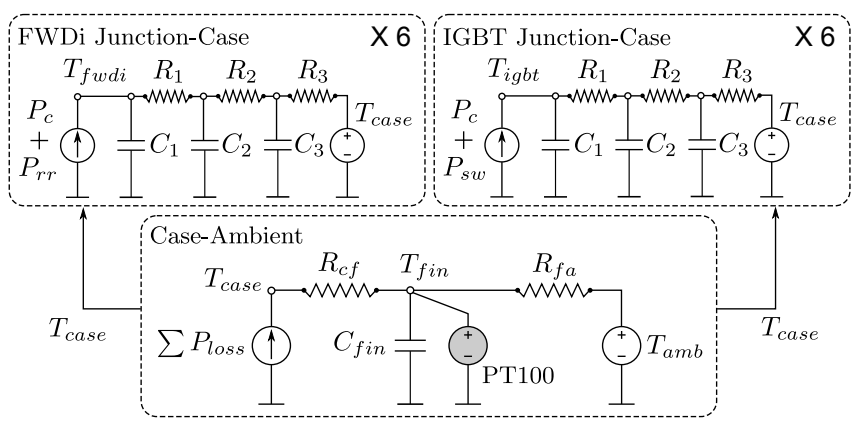

Fig. 7. Thermal networks for junction temperature estimation.
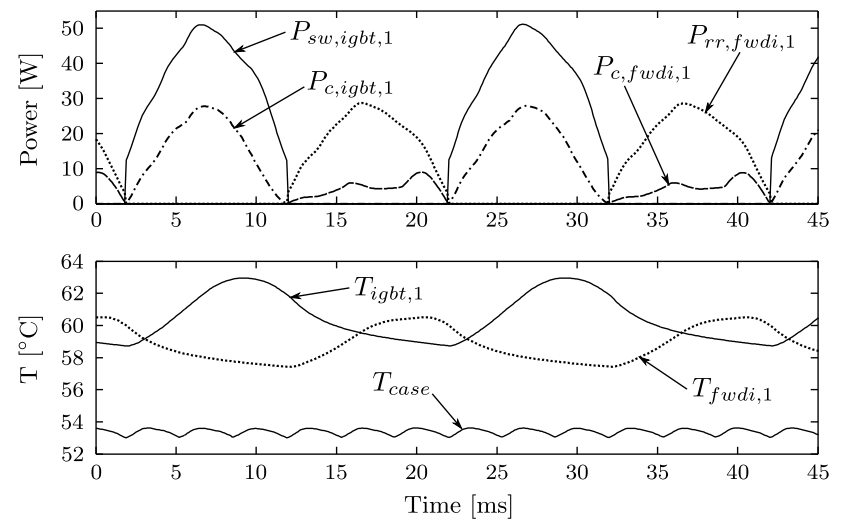

Fig. 8. Loss and thermal model output for IGBT-FWDi pair 1 at $67 \mathrm{Nm}$, $1000 \mathrm{RPM}, T_{s}=1 / 10000 \mathrm{~s}, U_{d c}=550 \mathrm{~V}$ and $T_{f i n}=50{ }^{\circ} \mathrm{C}$.

to limit parasitic effects (noise, harmonic losses) and maintain acceptable current control loop performance, switching frequency is never reduced below $4 \mathrm{kHz}$. A second control action calculates the maximum allowable IGBT or FWDI loss $P_{\text {loss }}^{*}$ according to the available temperature margin. The first term in (19) indicates the additional power loss, necessary to reach $T_{l i m}$ within a time $\tau_{c l}$ with $C$ the junction-case thermal capacity. The second term $\bar{P}_{\text {loss }, s s}$ reflects the heat dissipation which is estimated as the steady state device loss.

$$
P_{l o s s}^{*}=\frac{C}{\tau_{c l}}\left(T_{l i m}-T_{m e a s}\right)+\bar{P}_{l o s s, s s}
$$

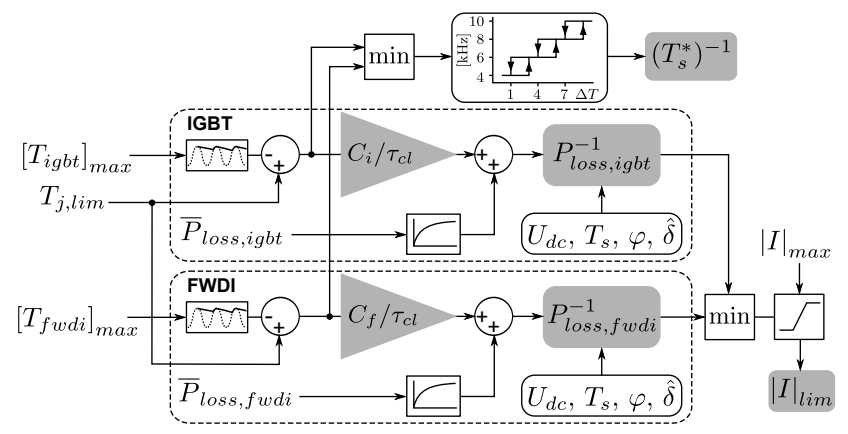

Fig. 9. Active thermal management by adaptive current limiting and switching frequency reduction. 


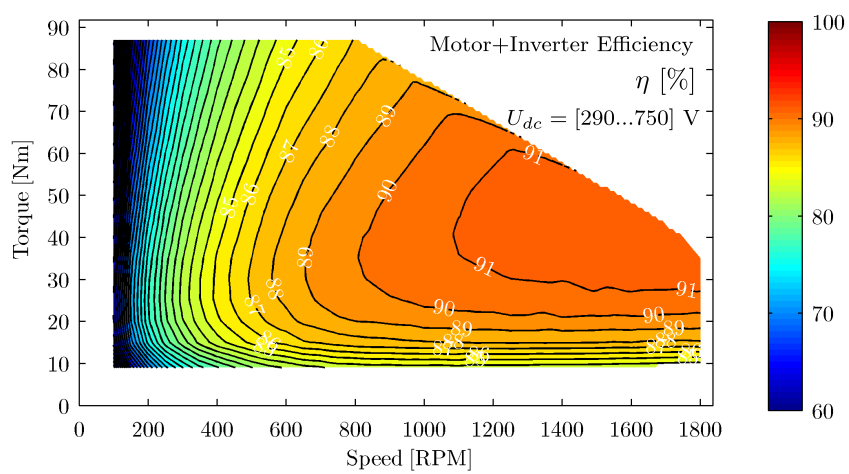

Fig. 10. Efficiency (motor and inverter) with a variable dc-link voltage.

Next, $P_{\text {loss }}^{*}$ is converted into a current limit with an inverse loss model. Finally, the most stringent current limit is selected and saturated between zero and an absolute maximum $|I|_{\max }$. The resulting $|I|_{\text {lim }}$ is transmitted to the constraint optimization loop which will limit torque, only if the actual current setpoint lies outside the dynamic current limit circle.

\section{EXPERIMENTAL RESULTS}

\section{A. Efficiency Assessment}

A first experiment assesses the influence of adaptive DClink voltage control on motor and inverter efficiency. Steady state power measurements on the DC-link, inverter output and motor shaft are carried out for a large number of speedtorque operating points (within the power range of the frontend converter) for a fixed $(550 \mathrm{~V})$ versus variable $(290-750 \mathrm{~V})$ bus voltage. Fig. 10 gives the recorded motor-inverter efficiency map with a variable DC-link voltage as shown in Fig. 5. Note that active frontend efficiency is not included. Compared to a fixed voltage, Fig. 11 displays the efficiency difference which is positive over the entire operating range and largest at low speed. Below 800 RPM, the bus voltage is set to the lower control bound of $290 \mathrm{~V}$ which implies a significant reduction of switching losses. Looking at the comparative loss map in Fig. 12, the saving is approximately $100 \mathrm{~W}$ at nominal torque and is independent of speed. Motor efficiency (not shown) is not noticeably affected in this region. Above 800 RPM the DC-link voltage is gradually increased (Fig. 5). Hence, the switching loss reduction compared to a fixed $550 \mathrm{~V}$ DC-bus becomes smaller. Around 1500 RPM, the difference is nearly zero as bus voltage in both cases are equivalent. Beyond this point, the fixed DC-link drivetrain goes into field-weakening operation yielding additional conduction and copper losses in the inverter and motor respectively. In contrast, the adaptive bus voltage strategy remains in MEPNm mode at high speed.

\section{B. Driving Cycle}

To study the proposed control strategy under realistic vehicle load conditions, a driving cycle experiment was conducted. The speed-controlled DC machine behaves as a dynamic load according to the following equation:

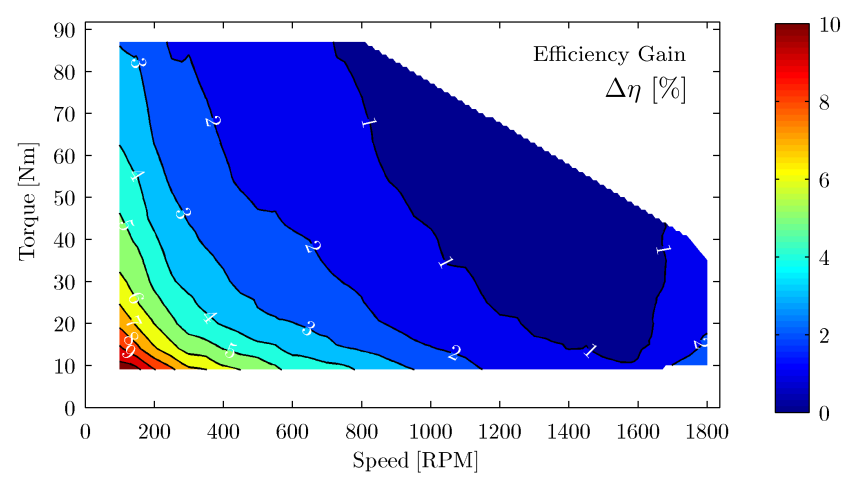

Fig. 11. Efficiency gain of the motor-inverter combination with a variable instead of a fixed dc-link voltage.

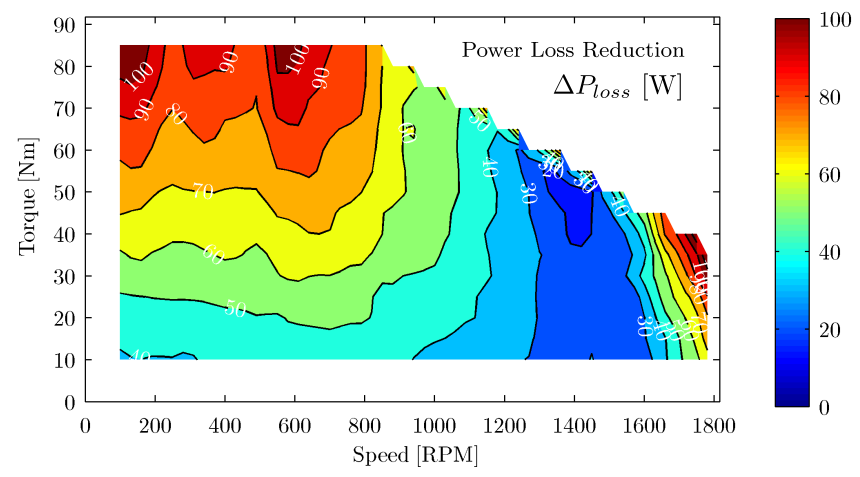

Fig. 12. Power loss reduction of the motor-inverter combination with a variable instead of a fixed dc-link voltage.

$$
T_{e l}=J \frac{d \omega}{d t}+\operatorname{sign}(\omega) \cdot C+\operatorname{sign}(\omega) \cdot D \omega^{2}
$$

with $T_{e l}$ the PMSM torque, $\omega$ the angular motor frequency, $J=5.5 \mathrm{~kg} \cdot \mathrm{m}^{2}$ the emulated inertia, $C=5 \mathrm{Nm}$ the Coulombfriction and $D=7.8883 e^{-4} \mathrm{Nm} /(\mathrm{rad} / \mathrm{s})^{2}$ the drag coefficient. The PMSM tracks the speed trajectory of a US06 driving cycle which results in the torque profile in Fig. 13. An identical test run is completed with a fixed and a variable DC-link voltage. Comparison of Fig. 14(a) and Fig. 14(b) reveals that the variable DC-link voltage control strategy is able to reduce peak power losses and hence also junction temperatures at low speed, high torque operating points. This type of load is typical for repeated acceleration and regenerative braking in urban traffic. Furthermore, avoiding field-weakening operation and the associated current-dependent losses at high speed reduces power loss as well. Over the entire US06 driving cycle, variable DC-link control yields a $7 \%$ reduction of energy loss. In terms of driving cycle energy consumption (measured at the DC link), this is only a $0.75 \%$ improvement. However, Fig. 12 suggest that a much larger saving can be obtained for low speed driving cycles, i.e. urban traffic, or very high speed trajectories. The US06 driving cycle mainly operates between 1000 and 1500 RPM where savings are small. This is because of the minor difference in DC-link voltage between both strategies in this area as shown in Fig. 5. 

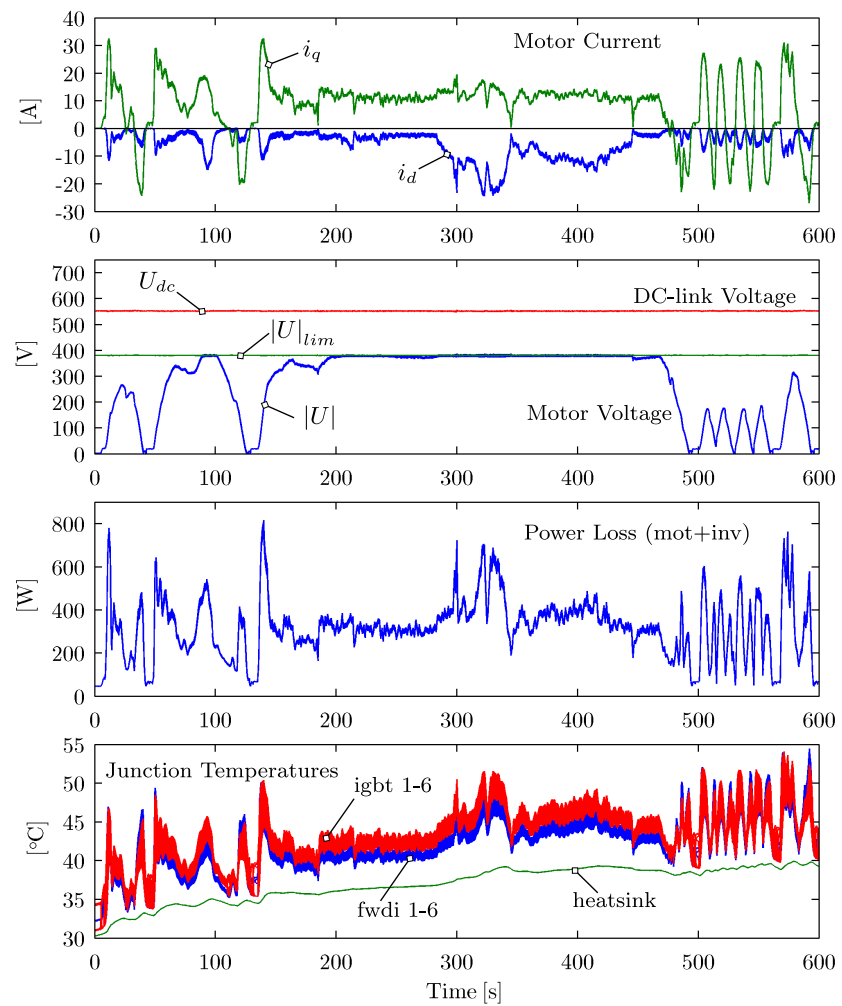

(a) Fixed DC-link voltage: $U_{d c}=550 \mathrm{~V}$
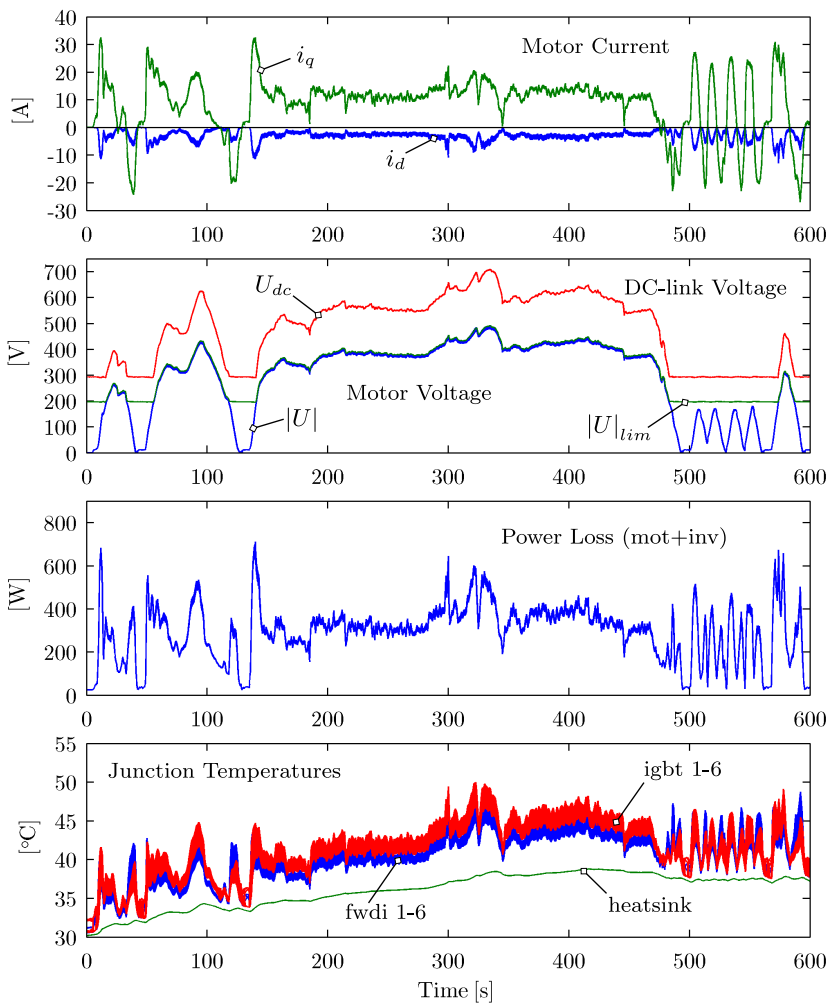

(b) Variable DC-link voltage: $U_{d c}=[290 \ldots 750] \mathrm{V}$

Fig. 14. US06 driving cycle speed trajectory tracking: Current, voltage, power loss and junction temperatures with a fixed versus variable DC-link voltage.
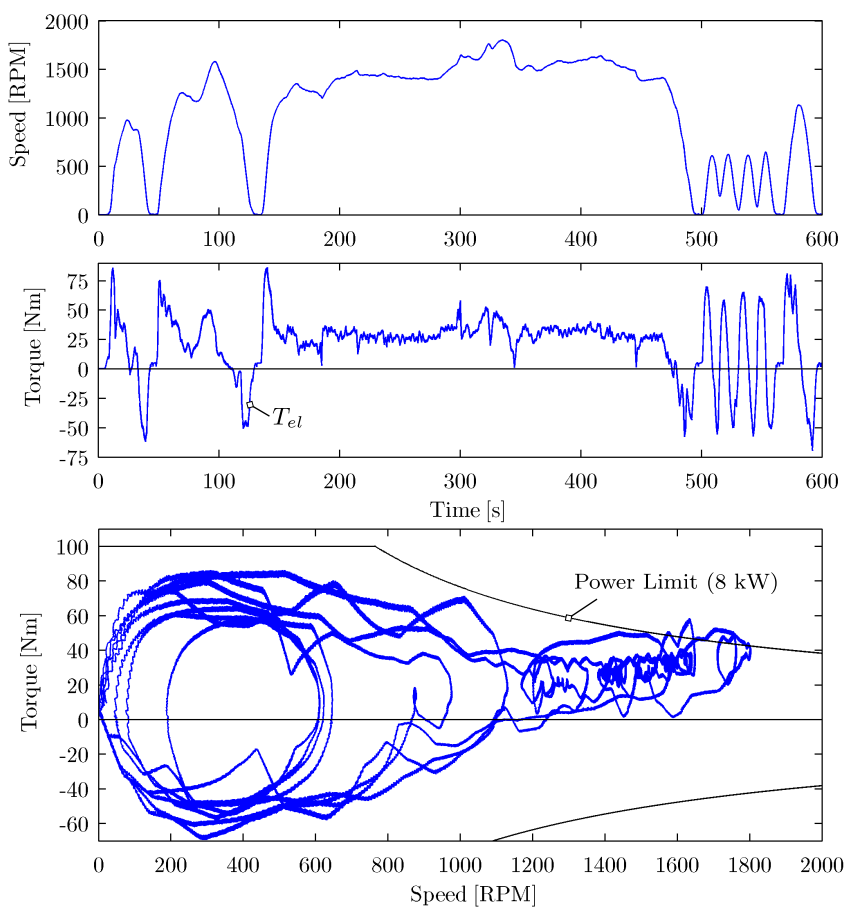

Fig. 13. US06 driving cycle speed trajectory tracking: Measured motor torque with dynamic load emulation according to (20).

\section{Thermal Management}

The previous experiments indicated that adaptive voltage control decreases switching losses considerably in low speed high torque operating points. Consequently, the IGBT and FWDI junctions will remain cooler and show less temperature cycling under heavy acceleration, benefiting lifetime and reliability. Vice versa, the maximum output torque for a given junction temperature limit will be higher with a variable DC-link voltage. This is illustrated with three comparative acceleration tests from 0 to 1000 RPM with an emulated load according to (20). Fig. 15(a) shows the result for a $550 \mathrm{~V}$ system with a fixed $|I|_{l i m}$ (no active thermal management). The drivetrain delivers the $80 \mathrm{Nm}$ torque request and accelerates to 1000 RPM in 8 seconds with a peak FWDI junction temperature of over $70{ }^{\circ} \mathrm{C}$. In Fig. 15(b), the active thermal management is activated with $T_{j, \text { lim }}$ set at $60{ }^{\circ} \mathrm{C}$. Due to the adaptive current limit $|I|_{\text {lim }}$, the $80 \mathrm{Nm}$ torque setpoint cannot be satisfied and the target speed is only reached after 16 seconds. Fig. 15(c) shows the same experiment, but now with variable voltage control. The lower DC-link voltage allows a larger $|I|_{\text {lim }}$, i.e. higher torque, for equal losses. Hence the 01000 RPM acceleration under the same thermal constraint is achieved in 10 seconds only. It should be noted that switching frequency adaptation was disabled during this test $(10 \mathrm{kHz})$. Enabling it would increase torque under thermal constraints even further, but this result was omitted from this paper. 

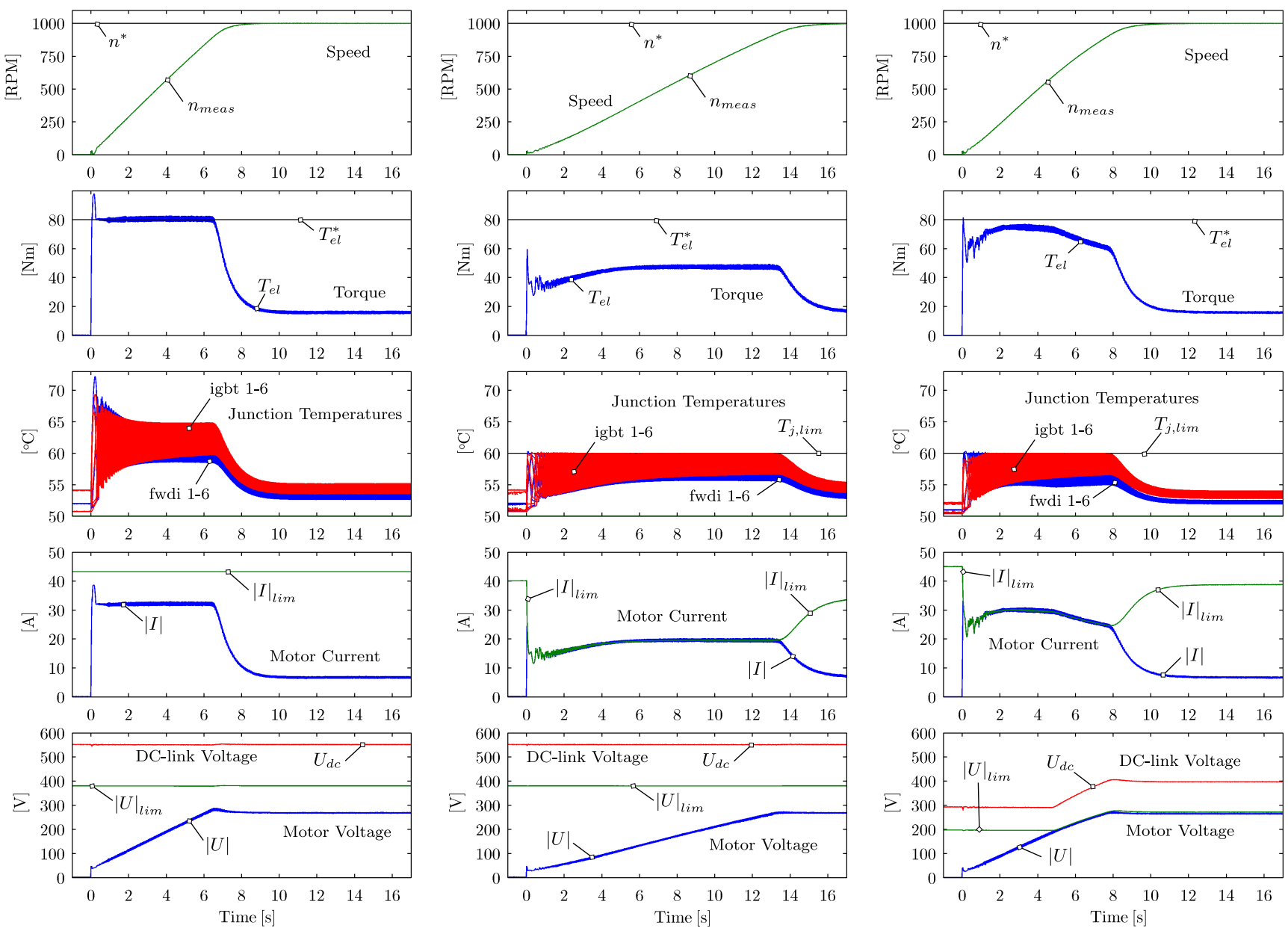

(a) $U_{d c}=550 \mathrm{~V}$ - Thermal management off

(b) $U_{d c}=550 \mathrm{~V}$ - Thermal management on

(c) $U_{d c}=[290 \ldots 750] \mathrm{V}-$ Thermal management on

Fig. 15. Acceleration test from 0 to 1000 RPM: Performance comparison under thermal constraints with a fixed versus variable DC-link voltage.

\section{CONCLUSION}

This paper investigated the potential benefits of a variable DC-link voltage control strategy, regarding thermal management of a series-hybrid drivetrain. By dynamically adapting the bus voltage to the requirements of the torque-speed operating point, motor and inverter losses are reduced significantly compared to a conventional fixed DC link. Next to an efficiency improvement, the reduction of inverter losses also implies lower switching device temperatures. This in turn facilitates a higher torque rating without jeopardizing reliability.

\section{REFERENCES}

[1] Y. Song and B. Wang, "Survey on reliability of power electronic systems," Power Electronics, IEEE Transactions on, vol. 28, no. 1, pp. 591-604, 2013.

[2] A. Wintrich, U. Nicolai, W. Tursky, and T. Reimann, "Application manual power semiconductors," SEMIKRON International, 2011.

[3] D. Hirschmann, D. Tissen, S. Schroder, and R. De Doncker, "Reliability prediction for inverters in hybrid electrical vehicles," Power Electronics, IEEE Transactions on, vol. 22, no. 6, pp. 2511-2517, nov. 2007.

[4] H. Huang and P. Mawby, "A lifetime estimation technique for voltage source inverters," Power Electronics, IEEE Transactions on, vol. 28, no. 8 , pp. 4113-4119, 2013.
[5] J. Lemmens, P. Vanassche, and J. Driesen, "Thermal management in traction applications as a constraint optimal control problem," in IEEE 2012 Vehicle Power and Propulsion Conf. (VPPC), 2012, pp. 36-41.

[6] C.-Y. Yu, J. Tamura, and R. Lorenz, "Control method for calculating optimum dc bus voltage to improve drive system efficiency in variable dc bus drive systems," in IEEE 2012 Energy Conversion Congress and Exposition (ECCE), sept. 2012, pp. 2992-2999.

[7] K. Yamamoto, K. Shinohara, and T. Nagahama, "Characteristics of permanent-magnet synchronous motor driven by pwm inverter with voltage booster,' Industry Applications, IEEE Transactions on, vol. 40, no. 4 , pp. $1145-1152$, july-aug. 2004.

[8] K. Yamamoto, K. Shinohara, and H. Makishima, "Comparison between flux weakening and pwm inverter with voltage booster for permanent magnet synchronous motor drive," in IEEE 2002 Power Conversion Conference - Osaka (PCC '02), 2002, pp. 161-166.

[9] M. Okamura, E. Sato, and S. Sasaki, "Development of hybrid electric drive system using a boost converter," Toyota Motor Corporation, 2003.

[10] J. Estima and A. Marques Cardoso, "Efficiency analysis of drive train topologies applied to electric/hybrid vehicles," Vehicular Technology, IEEE Transactions on, vol. 61, no. 3, pp. 1021-1031, march 2012.

[11] Triphase NV. 3PExpress ${ }^{\text {TM }}$, Rapid System Development for Power Conversion Applications. [Online]. Available: www.triphase.com

[12] "CM75TU-24F IGBT power module," Data sheet, Mitsubishi Electric.

[13] P. Vanassche and J. Lemmens, "PMSM Drive Control," White Paper (unpublished), Triphase NV, 2011.

[14] “COMBIVERT F5,” Instruction Manual, Karl E. Brinkmann GmbH 